\title{
Image Forgery Detection Algorithm Based on Non Sampling Wavelet Transform and Zernike Moments
}

\author{
Xiao Jinke ${ }^{1}$ \\ 1. Public Security Fourteen Bureau Zhuhai City Guangdong Province, \\ Zhuhai Guangdong, 519000 \\ 1036671926@qq.com
}

\begin{abstract}
In order to solve such problems as poor robustness and low detection accuracy in the present copying \& pasting forgery detection algorithm, an image copying \& moving forgery detection algorithm based on non-sampling wavelet transform (undecimated wavelet transform, UWT) coupling with Zernike moments is proposed in this article. Firstly, the non-sampling wavelet transform is adopted to decompose the input image into approximation coefficient $L L$ and detail coefficient $H H$ to obtain the similarity and the diversity of the image blocks; secondly, the overlapped blocks are divided to calculate the sub-block interval of the image; thirdly, the sub-blocks are ordered and classified according to LL similarity and HH diversity; fourthly, Zernike moments are introduced to construct the distance matrix for image feature matching and image forgery detection. The experiment shows: the algorithm proposed thereby has good translation invariance and amplitude rotation invariance, good post-processing resistance performance, high detection accuracy, low false negative rate and low false positive rate.
\end{abstract}

Keywords: Non sampling wavelet transform; Zernike moments; Copy \& paste; Image forgery detection; Robustness

\section{Introduction}

Along with the rapid development of science \& technology and Internet, digital images have become one of the most popular and influencing propagation modes. Due to the research and development of the powerful image processing software, people can easily tamper images and the tampered images are very vivid and can be rarely identified by human eyes, thus bringing great troubles to the subsequent use of images. As a result, image forgery detection becomes a crucial research field [12]. In the aspect of image forgery, the image copying \& moving forgery method becomes one of the most frequently used image tampering measures [3]. For example, $\mathrm{Wu}$, et al., have designed an image copying \& rotating forgery detection technology based on dual-tree complex wavelet transform, and meanwhile the experimental result has verified the algorithm rationality. This algorithm is based on similar image blocks for matching, so if the two detected image blocks have certain similarity, then the two image blocks will be falsely regarded as the copied \& moved zones and accordingly the real copied \& moved zones cannot be eliminated, thus causing the false detection possibility. Additionally, CHRISTLEI, et al., [5] have proposed the forgery detection algorithm based on image pixel matching, and relevant experiment has proven that this algorithm has reduced algorithm complexity and can well eliminate JPEG compression influence, thus to have high copied \& pasted zone location accuracy. However, if the copied \& pasted zones are located between two or among more than two image sub-blocks, the detection performance of this algorithm will be significantly reduced. Mahdian, et al., [6] 
have rationally assumed the zone copying tamper and designed a new image forgery detection technology, but this algorithm needs long detection time and cannot detect multiple forgery zones in the target image.

Therefore, an image copying \& moving forgery detection algorithm based on non-sampling wavelet transform (UWT) coupling with Zernike moments is proposed in this article. Specifically, Zernike moments are introduced to construct distance matrix for image feature matching and image forgery detection. Finally, the detection performance of the forgery location technology proposed in this article is also verified.

\section{Mathematical Model for Copying \& Moving Forgery}

Image copying \& moving forgery as a common forgery measure aims at copying a certain part of the original image and then pasting it to the other non-overlapped zones of the same image in order to conceal or add new contents. The forgery model thereof is simplified as follows [7]:

$$
I^{\prime}(x, y)=T[I(x, y)]+n(x, y)
$$

In the above formula, $I(x, y)$ refers to the original image, $I^{\prime}(x, y)$ refers to the forged image, $T$ refers to the operating function for $I(x, y)$, and $n(x, y)$ refers to random noise. The implementation process of the image copying \& moving forgery is as shown in Figure.1.

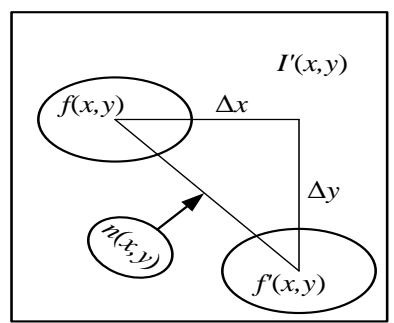

Figureure 1. Image Copying \& Moving Process

$f(x, y)$ is the copied zone of $I(x, y), f^{\prime}(x, y)$ is the pasted zone of $I^{\prime}(x, y)$, $f(x, y)$ and $I(x, y)$ shall meet the connectivity principle and keep a certain distance, without any overlap. $\Delta x, \Delta y$ are the movement displacements of the copied zone and the pasted zone, and such displacements are usually not smaller than the forgery zone. The copied zone is sourced from the same image, so the features thereof are much similar to those of the original image, but the copied zone is usually decorated through noise, fuzziness, JPEG lossy compression, etc. during the forgery process to cover the forgery marks, thus weakening the similarity of the copied zone and the pasted zone and bringing significant difficulty to the detection.

The image copying \& pasting forgery detection is mainly used to judge whether the image has the sub-blocks with large same or similar area, and mainly includes the following steps: 1. Image preprocessing; 2. Image block division; 3. Image block characteristic quantity extraction; 4. Image block matching; 5. Identification and location based on preset rules.

\section{Forgery Detection Algorithm}

Due to good time-frequency localization characteristics, wavelet transform (WT) is greatly popular in image processing field and signal analysis field. In mode identification, signal translation invariance is very important, and the relative value of the translated signal shall be also translated rather than altered. Discrete wavelet 
transform (DWT) can be used to obtain the image characteristic quantity through transformation, and the flexible translation can detail the signal in multiple scales, thus to well adapt to the signal analysis requirements and obtain the signal detail information. The down-sampling adopted by DWT can destroy the translation invariance of the wavelet transform and accordingly makes DWT become very sensitive to the initial position of the signal, so any tiny movement in time domain or space domain will cause the great variation of the wavelet transform coefficient[8].

For the detection technology based on non-sampling wavelet transform in this article, firstly, the image is decomposed through UWT in order to divide the overlapped blocks and respectively extract the low-frequency components (fuzzy information) LL and high-frequency components (detailed information) $\mathrm{HH}$; secondly, the similarity and the diversity of each group of blocks are calculated in order to obtain the distance of each group of blocks; thirdly, according to the distance obtained thereby, LL results are arranged by an ascending sequence and $\mathrm{HH}$ results are arranged by a descending sequence; fourthly, LL similarity and HH diversity are combined for relevant judgment, and meanwhile Zernike moments are introduced therein to construct the distance matrix for image feature matching and image forgery detection. The operation procedure of the algorithm proposed in this article is as shown in Figure 2.

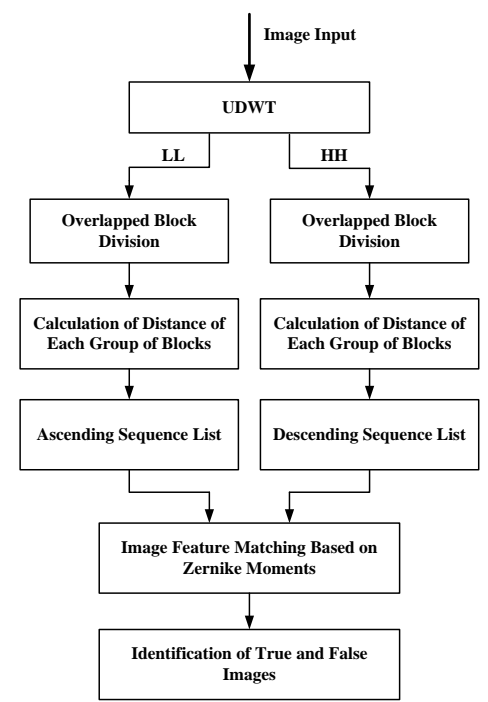

Figure 2. Algorithm Flow Chart

\subsection{Non Sampling Wavelet Transform}

For image identification, signal features must keep translation invariance in order to ensure that the signal features will be correspondingly translated rather than destroyed when an image zone is moved [9]. For example, for the image copying \& moving forgery, the two same zones of the copied zone and the pasted zone will not be destroyed. If the translation invariance cannot be kept, then the two zones will be differently represented and the missing detection may occur during the forgery detection process. In DWT, due to the existence of the down-sampling, the translation invariance is not kept; the wavelet coefficient is calculated according to formulae (1) and (2), and each direction factor is obtained through the downsampling convolution. The translation invariance is kept in DWT algorithm, so the tiny deviation of the input image may bring great influence to DWT coefficient in different scales and the tiny spatial deviation may generate different feature vectors to the copied and pasted zones. Meanwhile, DWT has pseudo Gibbs phenomenon 
and cannot provide ideal detection effect for such signal analysis as edge detection, denoising and texture analysis. In order to conquer the defects of DWT, UWT algorithm with translation invariance is proposed in this article, and this algorithm excludes down-sampling and wavelet coefficient reduction, so such wavelet transform is called non sampling wavelet transform.

If $\mathrm{I}$ is assumed as the input image, $h(k)$ and $g(k)$ are respectively assumed as low pass filter (LPF) and high pass filter (HPF), then UWT calculation of the image can be expressed as follows:

Initial condition: scale $j=0$ and $\mathrm{I}^{0}=\mathrm{I}$; the scaling proportion and the wavelet coefficient are respectively calculated at $j=1,2, \ldots, J$ according to formulae (2) and (3).

$$
\begin{aligned}
c^{j+1}(n) & =\sum_{k} h(k) c^{j}\left(n+2^{j} k\right) \\
d^{j+1}(n) & =\sum_{k} g(k) c^{j}\left(n+2^{j} k\right)
\end{aligned}
$$

$h^{j}(k)$ and $h^{j}(k)$ are respectively the filtered results of $h(k)$ and $g(k)$ at the scale $j$, and UWT process is as follows:

Initial conditions: $\mathrm{I}^{0}=\mathrm{I}$; the scaling proportion $I^{j}$ and the wavelet coefficient $D^{j}$ are calculated at $j=1,2, \ldots, J$.

$I^{j-1}$ is filtered in $h^{j-1}(k)$ filter.

$I^{j-1}$ is filtered in $g^{j-1}(k)$ filter.

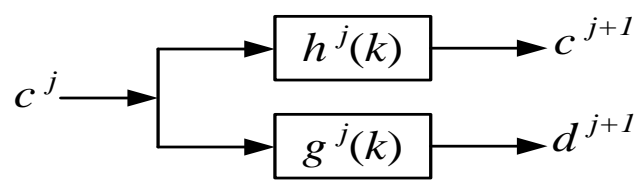

Figure 3. One-Dimensional Breakdown Drawing of uwt Algorithm

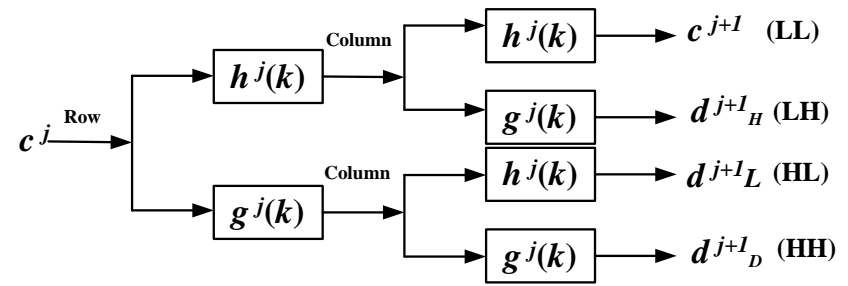

Figure 4. Two-Dimensional Breakdown Drawing of UWT Algorithm

Figure 3 shows the one-dimensional breakdown drawing of UWT algorithm. In wavelet transform, $I^{j}$ is called low pass sub-band (L) while $D^{j}$ is called high pass sub-band $(\mathrm{H})$. As a two-dimensional signal, an image is composed of four subbands, namely: LL, LH, HL and HH, wherein the size of each sub-band is as the same as that of the original image, as shown in Figure4. The two-dimensional image is decomposed through UWT along row and column in order to obtain low pass subband LL, horizontal high pass sub-band LH, vertical high pass sub-band HL and diagonal high pass sub-band $\mathrm{HH}$. 


\subsection{Zernike Moments}

After the image features are extracted through non sampling wavelet transform, it is necessary to find the corresponding relation of the image blocks for image matching calculation which is a key step in image identification. The common image gradation based algorithm cannot have ideal effect during image rotation and the image moment invariance can eliminate the adverse influence brought by image rotation, so Zernike moments with image moment invariance are adopted as the image matching algorithm ${ }^{[10]}$. The orthogonal set with integral unit circle is formed on the basis of the orthogonal polynomial $V_{n m}(x, y)$, and the model thereof is defined as follows:

$$
V_{n m}(x, y)=V_{n m}(r, \theta)=R_{n m}(r) e^{j m \theta}
$$

In the above formula, $(x, y)$ denotes the pixel point position; $n$ is the nonnegative integer and denotes the order; $m$ is the nonzero integer and denotes multiplicity; $r$ denotes radius and $\theta$ denotes angle, therein: $r=\sqrt{x^{2}+y^{2}}$ and $\theta=\operatorname{acr} \tan \frac{x}{y} ; R_{n m}(r)$, is Zernike radial polynomial and is defined as follows:

$$
R_{n m}(r)=\sum_{k=0}^{(n-|m|) / 2} \frac{(-1)^{k}(n-s) !}{k !\left[\frac{n+|m|}{2}-k\right] !\left[\frac{n-|m|}{2}-k\right]} r^{n-2 k}
$$

Therefore, n-order m-multiplicity two-dimensional Zernike moments can be defined as follows:

$$
Z_{n m}=\frac{n+1}{\pi} \iint_{x^{2}+y^{2} \leq 1} f(x, y) V^{*}{ }_{n m}(r, y) d x d y
$$

In the above formula, $*$ denotes complex conjugate. For a digital image, model (7) can be expressed in the polar coordinates as follows:

$$
Z_{n m}=\frac{n+1}{\pi} \sum_{r<10<\theta<2 \pi} \sum_{i}(r, \theta) V^{*}{ }_{n m}(r, \theta) r
$$

If the image is rotated by angle $\delta$, then Zernike moments are changed as:

$$
Z_{n m}=\frac{n+1}{\pi} \sum_{r<10<\theta<2 \pi} \sum_{n}(r, \theta) V^{*}{ }_{n m}(r, \theta-\delta) r
$$

After transformation, the following formula can be obtained:

$$
\begin{gathered}
Z_{n m}^{\prime}=Z_{n m} e^{j m \delta} \\
\left|Z_{n m}^{\prime}\right|=\left|Z_{n m}\right|
\end{gathered}
$$

According to models (9) and (10), Zernike moments of the image have amplitude rotation invariance and strong noise immunity. The specific steps for realizing Zernike moments are as follows:

Firstly, take a circle with the radius of $r$ for each image block of the image to be detected and then calculate Zernike moments of each feature neighborhood:

And then, construct distance matrix $D$, wherein the elements in matrix $D$ can be expressed as follows:

$$
D_{i j}=\sum_{m=1}^{n}\left|p_{i}-p_{j}\right|
$$

In the above formula, $P_{i}$ and $P_{j}$ are respectively the elements of the detected image. 
In the distance matrix $D$, respectively find the minimum values in row and column, wherein the features of $P_{i}$ and $P_{j}$ are matched when $D_{i j}$ gets the minimum values respectively in row and column at the same time.

\subsection{Specific Implementation}

In the forgery detection technology proposed in this article, firstly, the image is decomposed through UWT in order to obtain LL and HH, wherein LL is obtained through the low pass filters at the horizontal direction and the vertical direction and can be used to express the low-frequency components of the image, and LL subband is the approximate value of the image and has good effect for the repetitive identification; $\mathrm{HH}$ is obtained through the high pass filters at the horizontal direction and the vertical direction and includes high-frequency information mainly composed of noises and sharp corner angles, and $\mathrm{HH}$ sub-band can encode image noises but is distorted in forged image. The different expressions of the image forged by copying \& pasting at LL sub-band and HH sub-band in UWT algorithm is as shown in Figure6. Specifically, in Figure 6(a), the fish in the detected image is copied and pasted in the same image; the expression for the copied and pasted zones at LL is as shown in Figure 6(b); the expression for the copied and pasted zones at $\mathrm{HH}$ is as shown in Figure6(c). According to Figure6, the copied and pasted zones at $\mathrm{LL}$ are similar, and the copied and pasted zones at $\mathrm{HH}$ are distorted.

During forgery, the noise spectrogram of the image is distorted, and the forgery marks are tried to be concealed through edge smoothing or pasting addition for noise removal. Therefore, great difference exists during the processing of copying \& moving blocks in HH sub-band. Through the introduction of Zernike moments, the pixel point distance can be calculated according to model (12):

$$
D(p, q)=\sqrt{\frac{1}{N} \sum_{i=1}^{N}\left(p_{i}-q_{i}\right)}(
$$

In the above formula, $D(p, q)$ denotes the distance between block $p$ and block $q$; $p_{i}$ and $q_{i}$ denote the corresponding image gradations; $N$ denotes the total pixel values of the image block.

In LL, the calculated distance is stored in the ascending sequence list and the blocks with highest similarity are arranged at the top of the list. On the contrary, in $\mathrm{HH}$, the calculated distance is recorded in the descending sequence list, and the blocks with highest diversity are arranged at the top of the list. In order to eliminate rotation influence, Zernike moments based on the circle neighborhood of the feature points are adopted as the matching feature vectors to construct the distance matrix for image sub-block matching. If the distances of the two sub-blocks respectively at $\mathrm{LL}$ and $\mathrm{HH}$ are basically the same, then the zones are respectively the copied block and the moved block. Particularly, if the distance of one group of blocks $(p, q)$ is located at the nth position of LL ascending sequence list and is also in the range between the $n-1$ th position and the $n+1$ th position of HH descending sequence list, then this group of blocks are detected as the copied \& moved blocks.

Notably, for the original image probably having non-forged similar target, the similar targets in LL sub-band will be detected as copied \& moved zones and accordingly cause false detection. On the contrary, in HH sub-band, the missing detection may occur due to the diversity of the forged target. Therefore, LL and HH dual constraints are adopted to avoid false detection and missing detection so as to improve the detection accuracy of the algorithm. Meanwhile, the translation invariance of non sampling wavelet transform and the amplitude rotation invariance of Zernike moments are also adopted to improve the robustness and the noise immunity of the algorithm. 


\section{Experimental Result}

In order to verify the effectiveness and the superiority of the algorithm proposed in this article, two comparison groups are set: the algorithms proposed in literature [4] and literature [5] are respectively recorded as Algorithm A and Algorithm B. MATLAB8.0 is adopted to test the performance of the algorithm proposed in this article. Additionally, the simulation conditions are as follows: DELL I5, $2.3 \mathrm{GHz}$, four-core CPU, $500 G B$ hard disk, $4 G B$ memory, Windows 7 system. The image with the size of $384 \times 256$ is selected from CASIA TIDEV1.0 database [11].

In order to evaluate algorithm performance, false negative rate $\mathrm{FN}$, false positive rate $\mathrm{FP}$ and accuracy $\mathrm{AC}$ are introduced in this article [3].

\subsection{Copying \& Pasting Forgery Detection of the Tampered Zone without Rotation}

The image is forged by virtue of Photoshop CS3 software, as shown in Figure5. The original image is as shown in Figure 5(a); the image forged by copying \& pasting is as shown in Figure5(b); the detection result of the algorithm proposed in this article is as shown in Figure5(c), wherein the black zones respectively denote the copied zone and the pasted zone; the detection result of Algorithm A is as shown in Figure5(d), wherein the red zone is marked as the false detection zone; the detection result of Algorithm B is as shown in Figure5(e) and indicates that this algorithm has high false negative rate.

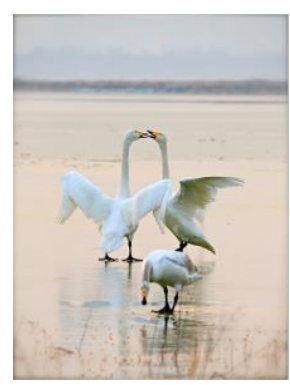

(a) Original image

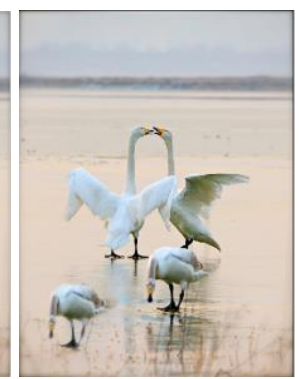

(b) Forged image

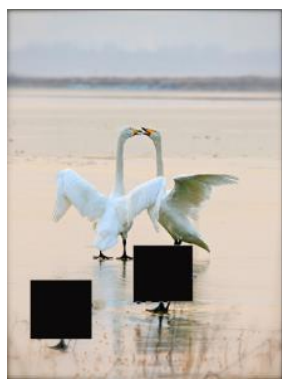

(c) Detection result of the algorithm proposed in this article

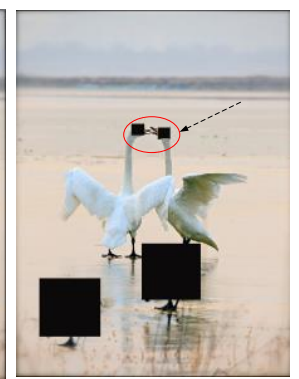

(d) Detection result of algorithm $A$

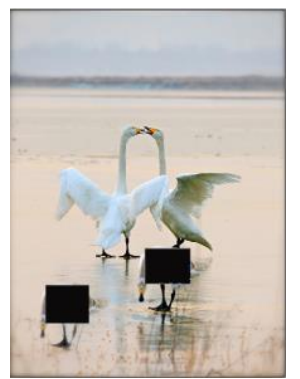

(e) Detection result of algorithm B

Figure 5. Detection Results of the Algorithm Proposed in the Article, Algorithm A and algorithm B

The copied image is totally divided into 384 image blocks with the size of $16 \times 16$, and the pasted image is similarly divided into 384 image blocks, totally 768 image blocks. Then, the above three algorithms are respectively adopted for image detection, and the detection results are as shown in Table 1. 
Table 1. Detection Results of the Algorithm Proposed in this Article, algorithm $A$ and algorithm $B$

\begin{tabular}{cccc}
\hline & $\begin{array}{c}\text { Algorithm Proposed in } \\
\text { this Article }\end{array}$ & Algorithm A & Algorithm B \\
\hline Accuracy AC (\%) & $735(95.70 \%)$ & $691(89.97 \%)$ & $629(81.90 \%)$ \\
False Positive Rate FP & 4.43 & 9.74 & 11.17 \\
$\begin{array}{c}(\%) \\
\text { False Negative Rate } \\
(\%)\end{array}$ & 6.43 & 11.72 & 14.31 \\
\hline
\end{tabular}

According to Table 1, the algorithm proposed in this article is superior to the other two algorithms in the aspects of accuracy, false positive rate and false negative rate. In detail, UWT with translation invariance is adopted for the algorithm proposed in this article, and the image is not destroyed in the copied and pasted zones, thus to well protect the detailed information of the tampered zone. The detection error measurement diagram for the three algorithms is as shown in Figure 7. According to Figure7, the false positive rate and the false negative rate of the algorithm proposed in this article are relatively lower than those of Algorithm A and Algorithm B.

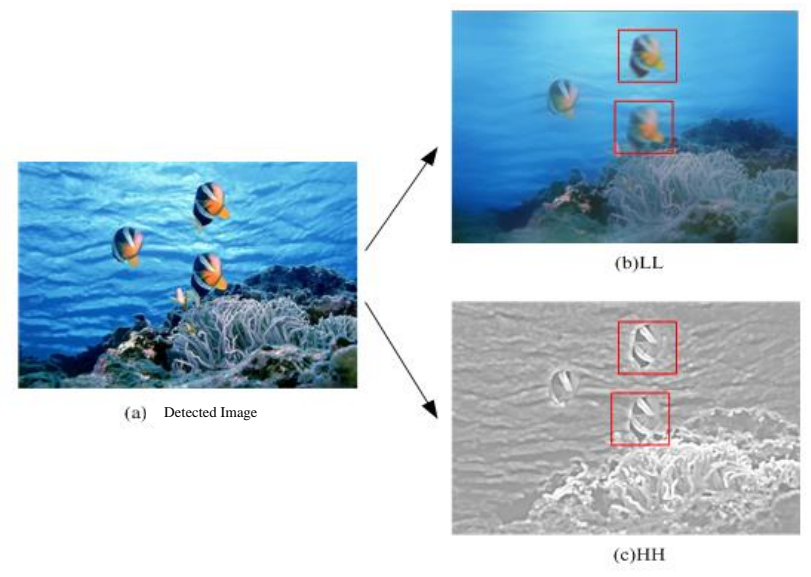

Figure 6. UWT Decomposition Result of the Image Forged by Copying \& Moving

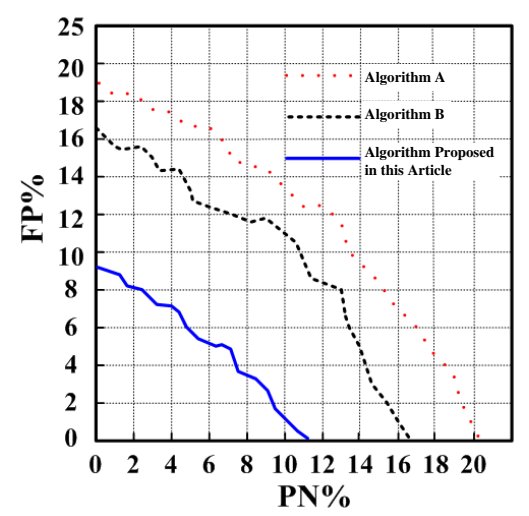

Figure 7. Detection Error Measurement Results of Three Algorithms 


\subsection{Test under Noise and JPEG Compression Factor}

200 images are randomly selected from CASIA TIDEV1.0 database for the test. For each image, a zone is selected to be copied and pasted to a non-overlapped zone, and then Gaussian noise or JPEG compression is implemented in the pasted zone, and finally the above three algorithms are respectively adopted for image test. The test results are as shown in Figure8, Figure9 and Figure10.
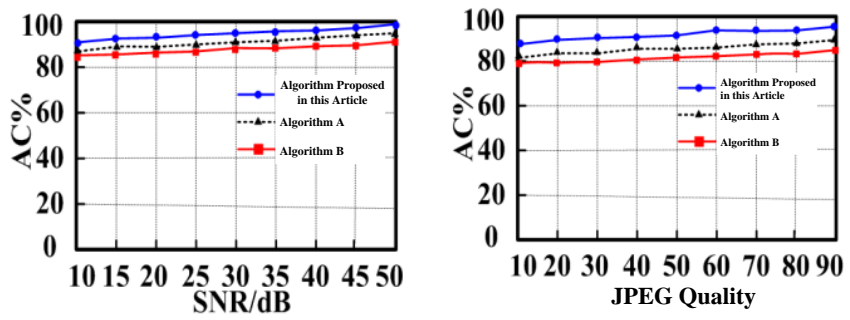

Figure 8. Detection Accuracy under Different Noises and JPEG Qualities
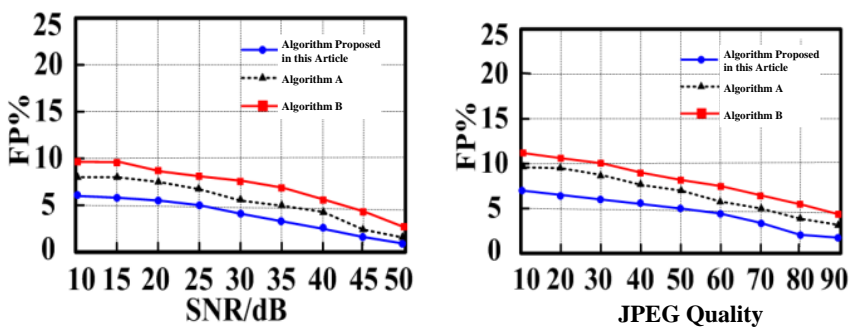

Figure 9. False Positive Rate under Different Noises and JPEG Qualities
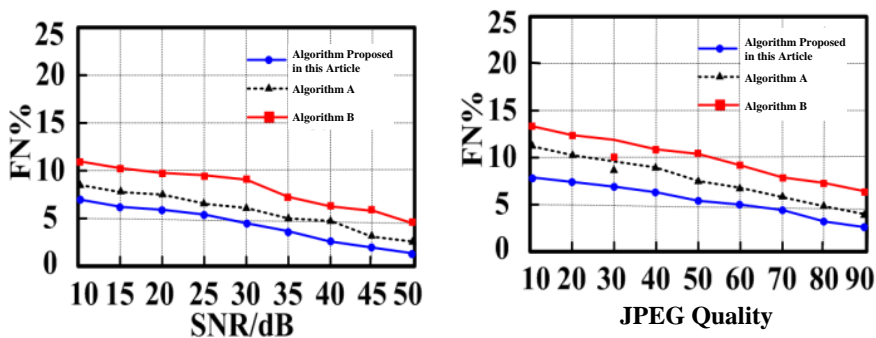

\section{Figure 10. False Negative Rate under Different Noises and JPEG Qualities}

According to Figure8, Figure9 and Figure10, for the same algorithm, if the tampered zone is less post-processed, then the detection accuracy thereof is higher while the false positive rate and the false negative rate thereof are lower; for the same post-processing, the algorithm proposed in the article is superior to Algorithm A and Algorithm B in the aspects of accuracy rate, false positive rate and false negative rate, thus indicating that the algorithm proposed in the article has good robustness, because LL and HH dual constraints are adopted for the algorithm proposed in the article to reduce false detection and missing detection of the image and improve the detection accuracy of the algorithm. In a word, the image forgery detection algorithm based on the translation invariance of non sampling wavelet transform and the amplitude rotation invariance of Zernike moments can improve algorithm robustness and has strong noise immunity. 


\section{Conclusion}

The image forgery detection technology based on non sampling wavelet transform coupling with Zernike moments is proposed in this article. Specifically, LL and $\mathrm{HH}$ sub-bands are obtained through non sampling wavelet transform to calculate the similarity and the diversity of the image blocks, and then LL similarity and $\mathrm{HH}$ diversity are combined for dual judgment. Meanwhile, Zernike moments are introduced therein to construct distance matrix for image feature matching. Finally, the images are selected from CASIA TIDEV1.0 database for algorithm evaluation, and the experimental result shows: the algorithm proposed in the article has the features of good noise immunity, good compression resistance, high algorithm accuracy, low false negative rate and low false positive rate.

\section{Reference}

[1] Jie He, Yishuang Geng, Yadong Wan, Shen Li, Kaveh Pahlavan, A cyber physical test-bed for virtualization of RF access environment for body sensor network, IEEE Sensor Journal, 13(10), 38263836, Oct. (2013).

[2] Wenhua Huang, Yishuang Geng, Identification Method of Attack Path Based on Immune Intrusion Detection, Journal of Networks, 9(4), 964-971, Jan. (2014).

[3] Guanqun Bao, Liang Mi, Yishuang Geng, Mingda Zhou, Kaveh Pahlavan, A video-based speed estimation technique for localizing the wireless capsule endoscope inside gastrointestinal tract, 2014 36th Annual International Conference of the IEEE Engineering in Medicine and Biology Society (EMBC), Aug. (2014).

[4] ] Degui Zeng, Yishuang Geng, Content distribution mechanism in mobile P2P network, Journal of Networks, 9(5), 1229-1236, Jan. (2014).

[5] Mingda Zhou, Guanqun Bao, Yishuang Geng, Bader Alkandari, Xiaoxi Li, Polyp detection and radius measurement in small intestine using video capsule endoscopy, 2014 7th International Conference on Biomedical Engineering and Informatics (BMEI), Oct. (2014).

[6] Gan Yan, Yuxiang Lv, Qiyin Wang, Yishuang Geng, Routing algorithm based on delay rate in wireless cognitive radio network, Journal of Networks, 9(4), 948-955, Jan. (2014).

[7] Guanqun Bao, Liang Mi, Yishuang Geng, Kaveh Pahlavan, A computer vision based speed estimation technique for localiz ing the wireless capsule endoscope inside small intestine, 36th Annual International Conference of the IEEE Engineering in Medicine and Biology Society (EMBC), Aug. (2014).

[8] Xinchao Song, Yishuang Geng, Distributed community detection optimization algorithm for complex networks, Journal of Networks, 9(10), 2758-2765, Jan. (2014).

[9] Jinyu Hu and Zhiwei Gao. Distinction immune genes of hepatitis-induced heptatocellular carcinoma[J]. Bioinformatics, (2012)., 28(24): 3191-3194.

[10] Jinyu Hu, Zhiwei Gao and Weisen Pan. Multiangle Social Network Recommendation Algorithms and Similarity Network Evaluation[J]. Journal of Applied Mathematics, 2013 (2013).

[11] Jinyu Hu and Zhiwei Gao. Modules identification in gene positive networks of hepatocellular carcinoma using Pearson agglomerative method and Pearson cohesion coupling modularity[J]. Journal of Applied Mathematics, 2012 (2012).

[12] Zhang, Xu. Spike-based indirect training of a spiking neural network-controlled virtual insect. 2013 IEEE 52nd Annual Conference on Decision and Control (CDC). IEEE, (2013).

[13] Wang, Ke. "Towards Scalable Distributed Workload Manager with Monitoring-Based Weakly Consistent Resource Stealing." (2015).

[14] Zhihan Lv, Tengfei Yin, Yong Han, Yong Chen, and Ge Chen. WebVR — web virtual reality engine based on P2P network. Journal of Networks. 6, no. 7 (2011): 990-998.

[15] ] Jiachen Yang, Bobo Chen, Jianxiong Zhou, Zhihan Lv. A portable biomedical device for respiratory monitoring with a stable power source. Sensors. (2015).

[16] Shuping Dang, Jiahong Ju, Matthews, D., Xue Feng, Chao Zuo. Efficient solar power heating system based on lenticular condensation. Information Science, Electronics and Electrical Engineering (ISEEE), 2014 International Conference on . 26-28 April (2014).

[17] Xiaolei Zhang, Yong Han, Dongsheng Hao and Zhihan Lv. ARPPS : Augmented Reality Pipeline Prospect System. 22th International Conference on Neural Information Processing (ICONIP 2015), Istanbul, Turkey. In press.

[18] Wang, Ke. "Overcoming Hadoop Scaling Limitations through Distributed Task Execution."

[19] Zhang, Su, Xinwen Zhang, and Xinming Ou. "After we knew it: empirical study and modeling of costeffectiveness of exploiting prevalent known vulnerabilities across iaas cloud." Proceedings of the 9th ACM symposium on Information, computer and communications security. ACM, (2014). 
[20] Wei Gu, Zhihan Lv, Ming Hao. Change detection method for remote sensing images based on an improved Markov random field. Multimedia Tools and Applications. (2016).

[21] ] Zhihan Lu, Chantal Esteve, Javier Chirivella and Pablo Gagliardo. A Game Based Assistive Tool for Rehabilitation of Dysphonic Patients. 3rd International Workshop on Virtual and Augmented Assistive Technology (VAAT) at IEEE Virtual Reality 2015 (VR2015), Arles, France, IEEE, (2015).

[22] Zhanwei Chen, Wei Huang, Zhihan Lv. Uncorrelated Discriminant Sparse Preserving Projection Based Face Recognition Method. Multimedia Tools and Applications. (2016).

[23] ] Zhihan Lv, Alaa Halawani, Shengzhong Feng, Haibo Li, and Shafiq Ur Rehman. 2014. Multimodal Hand and Foot Gesture Interaction for Handheld Devices. ACM Transactions on Multimedia Computing, Communications, and Applications (TOMM). 11, 1s, Article 10 (October 2014), 19 pages.

[24] Kaijun Leng, Wen Shi, Jinbo Chen, Zhihan Lv. Designing of a I-shaped less-than-truckload cross-dock: A simulation experiments study. International Journal of Bifurcation and Chaos. (2015).

\section{Authors}

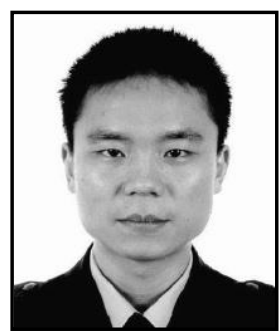

Xiao Jinke, (1981 -), male (Han nationality), He was born in Yiyang Hunan. Graduate from The Peking University, He researched on identified by the Ministry of public security, national public security contest third prize winner. He has traveled to Xinjiang aid forensics technology research and training work carried out, mainly engaged in information security, data recovery research. 
International Journal of Security and Its Applications

Vol. 10, No. 2 (2016) 